\title{
Analysis of periodic spectral gaps observed in the tuning range of free-electron lasers with a partial waveguide
}

\author{
R. Prazeres, ${ }^{*}$ F. Glotin, and J.-M. Ortega \\ LCP/CLIO Bâtiment 201P2, Université de Paris Sud, 91405 Orsay Cedex, France
}

(Received 22 April 2008; published 29 January 2009)

\begin{abstract}
A phenomenon of "spectral gaps" is observed in far-infrared free-electron lasers using partial waveguiding: the laser power falls down to zero at some particular wavelengths, whatever the beam adjustments are. We show that this effect is related to a periodic increase of the cavity losses, with a period depending on the dimensions of the waveguide. A code including diffraction effects exhibits good agreement with the measurements.
\end{abstract}

DOI: 10.1103/PhysRevSTAB.12.010701

PACS numbers: 41.60.Cr, 42.55.-f, 42.60.- v, 42.79.Gn

\section{INTRODUCTION}

Several infrared free-electron lasers (FELs) are presently used around the world as light sources by a large scientific community [1]. FELs are especially efficient in infrared since they produce high power laser pulses, and are tunable across a large wavelength range, typically 1 to 2 decades. The CLIO FEL [2] spans from 3 to $150 \mu \mathrm{m}$, obtained by varying the beam energy from 50 to $12 \mathrm{MeV}$. This is the largest tuning range obtained with a single beam line. However, as the wavelength increases, the diffraction limited optical mode transverse size tends to undergo losses at the undulator vacuum chamber (Fig. 1). The size of this chamber cannot be increased if one wants to maintain a sufficiently strong undulator magnetic field on axis. Therefore, several FELs $[3,4]$ have designed the vacuum chamber so as to use it as an optical waveguide in the direction parallel to the magnetic field. In this configuration, detailed measurements of FEL power at large wavelength exhibit a phenomenon of spectral gap: for example, at $\lambda=45 \mu \mathrm{m}$ the CLIO FEL power falls down to zero or vanishes strongly. This effect is always present, independently of electron beam tunings or of cavity mirror radius of curvature. This phenomenon is also observed in other infrared FELs, which are working in the same configuration: FELIX [3] and ELBE [4].

In order to provide an explanation for these spectral gaps, we have developed an in-house numerical code $[5,6]$ which calculates the cavity losses, the intracavity laser power of the FEL and the output laser power. This code takes into account the configuration of the optical cavity for the partially waveguided FEL with hole extraction. Finally, we show that a simple analytical calculation of the phase advance of light in the waveguide accounts for the periodic behavior of this spectral gap.

*rui.prazeres@u-psud.fr

\section{OPTICAL CAVITY}

The optical cavity of a FEL is always several meters long, because it includes enough space for the undulator and for the magnetic elements used to drive the electron beam. As an example, the layout of the CLIO FEL is displayed on Fig. 1. The distance between cavity mirrors is 4.8 meters. The vertical inner size of vacuum chamber is limited to $14 \mathrm{~mm}$, in order to allow wavelength tuning by the undulator gap. The horizontal size is $35 \mathrm{~mm}$. In the first approximation, the elliptical shape of the cross section of the waveguide allows one to consider the waveguide as a pair of infinite parallel plates, as shown in Fig. 1. A waveguide is created by using an undulator vacuum chamber of good optical quality. This is realized by using extruded aluminum which possesses a good conductivity and reflectivity.

Therefore, optical guiding only occurs along one axis corresponding to the minimum waveguide dimension (vertical axis in Fig. 1), whereas wave propagation is free along the other axis (horizontal in Fig. 1). Also, the waveguide length does not fill the whole space of the cavity: The area close to the mirrors is in "free propagation mode." This hybrid configuration induces a "partial guiding" of the laser mode. At short wavelength $(\lambda<10 \mu \mathrm{m})$ the waveguide does not modify the laser mode, because it is larger than its dimensions. The Gaussian beam propagation analytical theory is then valid. However, at large wavelength (typically $\lambda>50 \mu \mathrm{m}$ ) the laser mode becomes guided in the vacuum chamber, and remains free propagating elsewhere. In the intermediate regime, the mode is partially guided in the extremities of the waveguide.

Another feature of the cavity can be responsible for important perturbations of the laser mode: the output coupling is performed by a hole of 2 or $3 \mathrm{~mm}$ in the center of one cavity mirror. This solution is advantageous as compared to using a beam splitter because it does not create optical absorption, especially at large wavelength where 


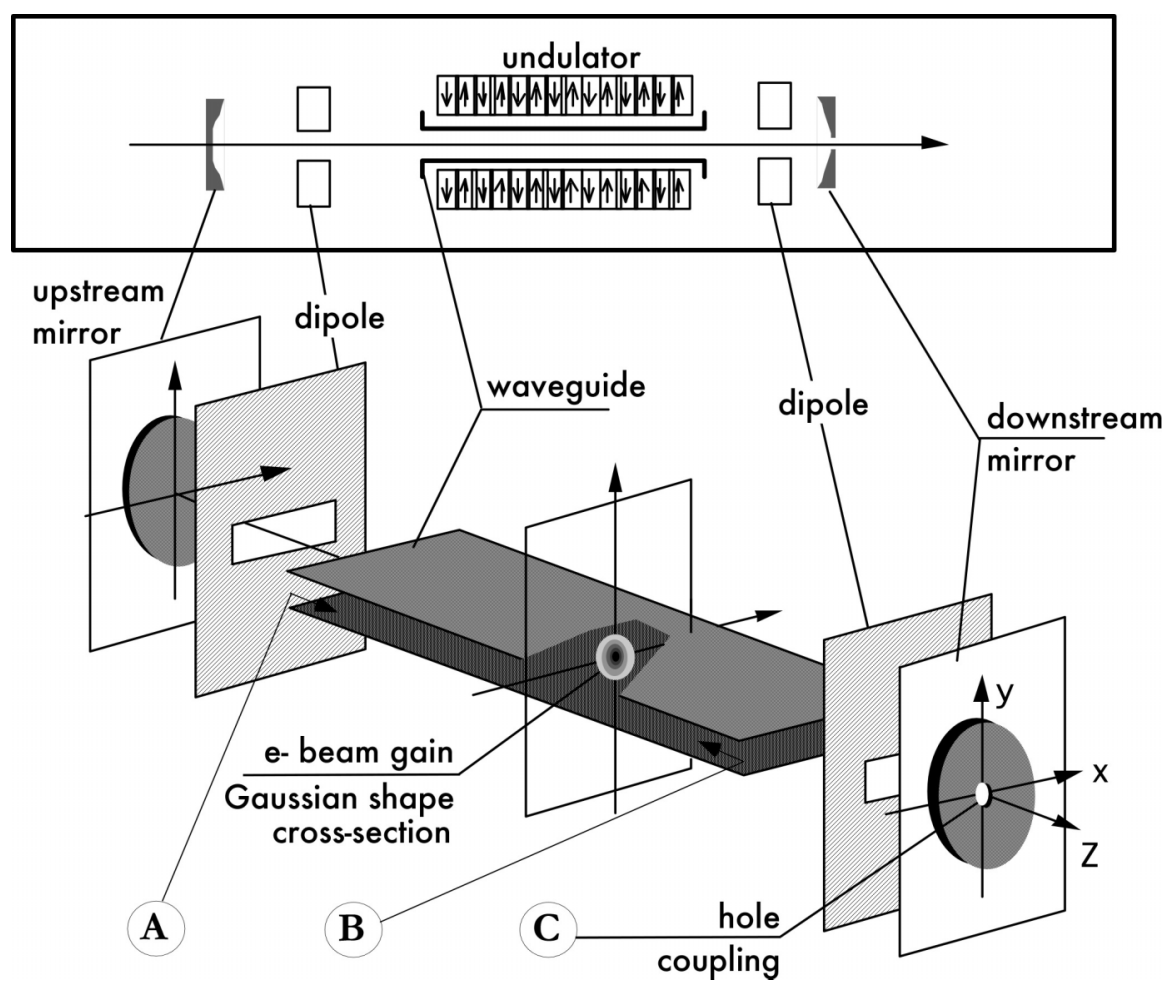

FIG. 1. Schematic layout of the CLIO free-electron laser optical cavity.

transparent materials are rare and of poor optical quality. However, the output coupling factor of the hole, i.e., ratio between output power and intracavity power, is strongly dependent on the transverse distribution of the laser mode at the output mirror.

A less important feature must still be considered here, which is typical of the FEL process, and is taken into account in the code (see Sec. III): the optical gain of the FEL is limited in space by the electron beam cross section, whose rms dimensions are close to 1 or $2 \mathrm{~mm}$. This influences the laser mode size in the undulator, as it induces a narrowing of the transverse profile of the laser.

In principle, the standard configuration for the cavity mirrors is a pair of spherical mirrors. However, at large wavelength, $\lambda>50 \mu \mathrm{m}$ for CLIO, a better configuration is to use toroidal mirrors, which refocus the beam at the entry and output of the chamber in the vertical direction, whereas on the other axis, the free propagation requires the standard radius of curvature. Toroidal mirrors allow to lase at $\lambda>100 \mu \mathrm{m}$ at CLIO. This toroidal configuration is used also at FELIX and ELBE.

\section{NUMERICAL CODE}

\section{A. General description}

The numerical code which has been used here $[5,6]$ calculates the propagation of the laser wave front $A(x, y)$ in the optical cavity. It uses an iterative process of wave propagation in the cavity, which converges to a steady state laser mode, corresponding to the FEL saturation regime. It takes into account the design of the cavity (mirrors dimensions and radius of curvature), including the waveguide effect in the undulator section, and the hole coupling in the output mirror. The gain coefficient is calculated in order to compensate the cavity losses at saturation. The operator of FEL gain is detailed below: it uses a special process in the case of waveguide environment. The calculation of the power [6] at laser saturation is based on the theory of FEL intensity saturation which has been developed by Dattoli et al. [7]. It takes into account the nonlinear behavior of small signal gain in high gain regime, it includes the inhomogeneous broadening of filling factor and the longitudinal mode coupling factor. It is a single wavelength theory, which does not include short pulse effects of the FEL and is limited to narrow linewidth FEL operation.

This code gives the amplitude distribution $A(x, y)$ of the laser mode at saturation, in any point of the cavity and all related parameters: the cavity losses $L$, the extraction rate $T_{X}$ of hole coupling, the intracavity laser power $P_{\text {in }}$, and the output power $P_{\text {out }}$. The cavity losses are equal to $L=$ $\Delta P_{\text {in }} / P_{\text {in }}$, where $\Delta P_{\text {in }}$ is the power loss of the intracavity laser pulse after one cavity round-trip. The extraction rate $T_{X}=P_{\text {out }} / P_{\text {in }}$ is the ratio between output power $P_{\text {out }}$ and intracavity power $P_{\text {in }}$.

\section{B. Mode selective gain operator in the waveguide}

The gain operator $(G)$, used in the code, is applied to input amplitude distribution $A(x, y)$ which is propagating in 
the center of the undulator waveguide. This amplitude may be decomposed into a sum $A=\Sigma\left(A_{q}\right)$ of eigenmodes $A_{q}(x, y)$ : only one mode number $q$ is used here because guiding only occurs along one axis. The FEL resonance wavelength $\lambda_{R(q)}$ is dependent on the phase velocity in the waveguide, which depends on the eigenmode number $q$ of the laser mode. Therefore, the spectral range of FEL gain is shifted by a quantity depending on $q$. As a consequence, the gain coefficient also depends on eigenmode number [8], and it may be close to zero when the laser wavelength is out of resonance $\lambda_{R(q)}$. The relative frequency shift between the first and third order mode is approximately equal to $\left[\lambda_{R(q=3)}-\lambda_{R(q=1)}\right] / \lambda_{R}=\lambda_{R} \lambda_{o} / b^{2}$, where $\lambda_{R}$ is the resonance wavelength without waveguide, $\lambda_{o}$ is the undulator period and $b$ is the small dimension of the waveguide. For CLIO this means about $1 \%$ at $45 \mu \mathrm{m}$, so just within the bandwidth, but for FELIX it is more than $3 \%$ at $53 \mu \mathrm{m}$. Therefore, in the worse case, only the fundamental mode TE1 and TM1 would be amplified, the other modes may be too far from undulator resonance.

The gain process used in our code takes into account such eigenmode filtering. The fundamental mode component $A_{q=1}(x, y)$ is calculated from input profile $A(x, y)$, and is amplified by the gain transverse distribution $G(x, y)$. This is calculated by the product $A^{\prime}=A_{1}[1+G(x, y)]$. However, since the gain transverse distribution $G(x, y)$ is not uniform, then the amplified mode $A^{\prime}$ includes high order modes: $A^{\prime}=\Sigma\left(A_{q}{ }^{\prime}\right)$. Therefore, the final output profile $A^{\prime \prime}=[G] A$ is a sum of the amplified fundamental mode $A^{\prime}$ and the initial sum of high order modes $\left(A-A_{1}\right)$. As a consequence, the amplification occurs on fundamental mode $A_{1}(x, y)$, but energy is still coupled to higher order modes.

In addition, during wave propagation in the optical cavity, the transition from free space to waveguide also induces an important coupling between fundamental and high order modes. Simulations have shown that such energy rearrangement, due to the coupling, takes place very rapidly in the cavity (less than one round-trip). As a consequence, in spite of the selective amplification of fundamental mode, the mode profile in the waveguide, at saturation, may contain an important amount of high order modes. The numerical simulation shows that the final result does not make a large difference when using the "mode selective" gain process (i.e. using exclusive amplification of eigenmode $q=1$ ) or uniform "standard" gain (i.e. using same rate of amplification for all eigenmodes). In order to have a quantitative estimation of the influence of this effect, we always display, in the following simulations, the results using both configurations of gain process. The real case corresponds to an intermediate solution between these extreme configurations. However, it is not possible to find the exact rate of amplification for each eigenmode, because the gain spectrum at saturation is not known in advance and is different from the well-known small signal gain spectrum.

\section{RESULTS FOR “CLIO”}

\section{A. Power measurements}

We have measured the output laser power of CLIO as a function of wavelength for various combinations of cavity mirrors. Wavelength tuning is made by undulator gap variation, whereas the electron beam energy is kept constant at $15 \mathrm{MeV}$. These measurements have been recorded in a purged chamber in order to avoid laser absorption in air.

The first set of measurements is using spherical mirrors and is displayed in Fig. 2. A spectral gap appears at $\lambda=$ $45 \mu \mathrm{m}$ and corresponds to a power decrease by a factor of about 2 . The simulation fits very well with measurements, including the absolute value of the power, with a realistic set of electron beam parameters: bunch charge $Q=$ $0.5 \mathrm{nC}$, energy spread $\sigma \gamma / \gamma=0.5 \%$, pulse length $\sigma z=$ $4 \mathrm{ps}$, emittance $150 \pi \mathrm{mm}$ rad. The red curve has been calculated with gain amplification exclusively on fundamental TE1 and TM1 modes. As described on Sec. III B, the energy on higher modes is supplied by coupling interaction between the modes. The blue curve corresponds to uniform amplification for all eigenmodes, i.e., the standard FEL gain process like in free space.

Figure 3 corresponds to a second set of measurements with a hybrid cavity, i.e., using a spherical upstream mirror and a toroidal downstream mirror. The spectral gap is more noticeable in this case. When using a full toroidal cavity, i.e., toroidal on both cavity mirrors, the power gap remains

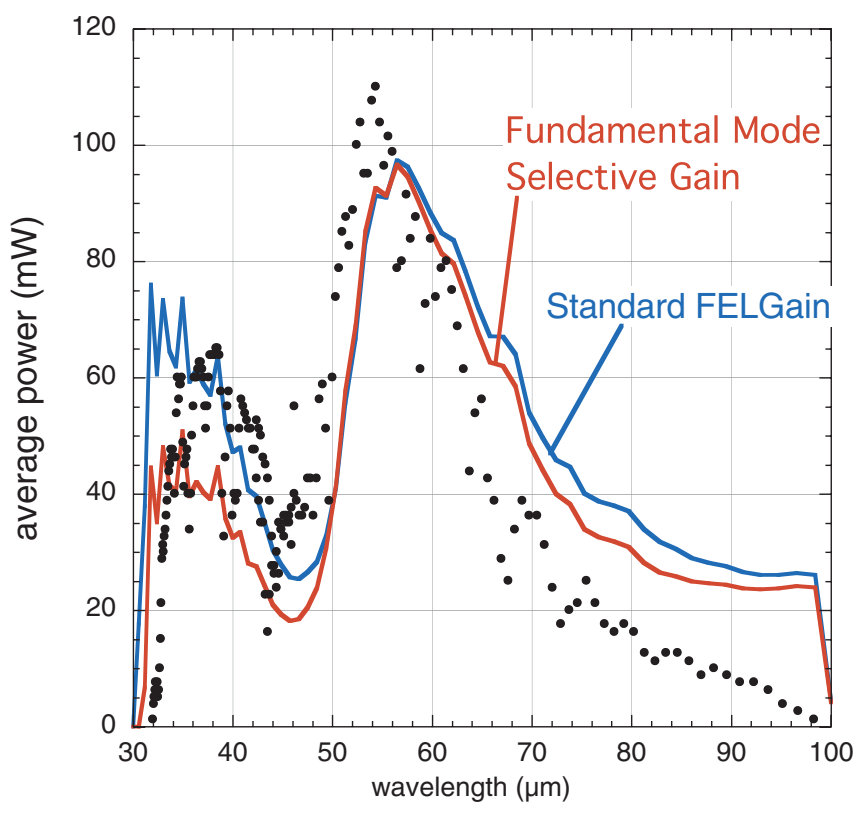

FIG. 2. (Color) Laser power vs centroid wavelength, for spherical mirrors cavity, using hole coupling of $2 \mathrm{~mm}$ (dotted line: measurement; continuous line: simulation). 


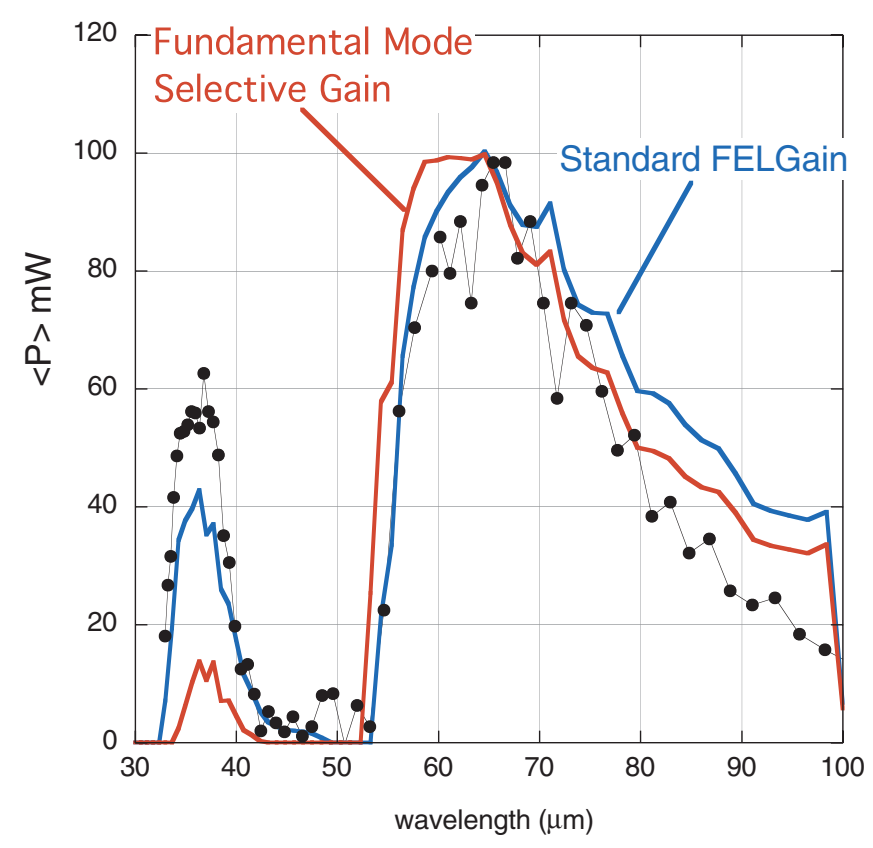

FIG. 3. (Color) Laser power vs centroid wavelength, for hybrid cavity: spherical for upstream and toroidal for downstream, using hole coupling of $3 \mathrm{~mm}$. Bold lines are simulations.

as shown in Fig. 4. These measurements show that spectral gaps are an intrinsic effect, independent from optical cavity configuration.

\section{B. Simulation for spherical cavity}

In order to explain the spectral gap phenomenon, we used the numerical code to compute the cavity losses $L$ and the hole coupling extraction rate $T_{X}$ as a function of wavelength, and the laser mode profile at various places in the optical cavity. Figures 5 and 6 display them in the case of spherical mirrors cavity, i.e., related to power variations in Fig. 2. The result is independent of the gain process (mode selective gain or uniform gain).

The reason of the spectral gap is clearly visible: the extraction rate $T_{X}$ exhibits a minimum at $\lambda=45 \mu \mathrm{m}$ (point $\lambda_{a}$ ). This is the first reason which explains the output power decrease at this wavelength.

Figure 6(a) displays the transverse profile of the laser mode, at the output mirror (point $\mathrm{C}$ in Fig. 1), for wavelengths $\lambda_{a}$ and $\lambda_{c}$. It is wider and exhibits a small minimum of intensity in the center for $\lambda_{a}$, which accounts for the decrease of $T_{X}$, which is the integral of the intensity across the hole.

However, this is not the only cause of spectral gaps: despite the lower extraction rate, the cavity losses are also maximum at $48.4 \mu \mathrm{m}$ (point $\lambda_{b}$ ). Mirror reflectivity is independent of wavelength, and Ohmic losses in the waveguide are negligible. A detailed analysis of the laser mode profile at the waveguide entrances, displayed in Fig. 6(b), shows that high losses in point $\lambda_{b}$ are due to a broadening of the transverse size of laser mode, which does not fit the

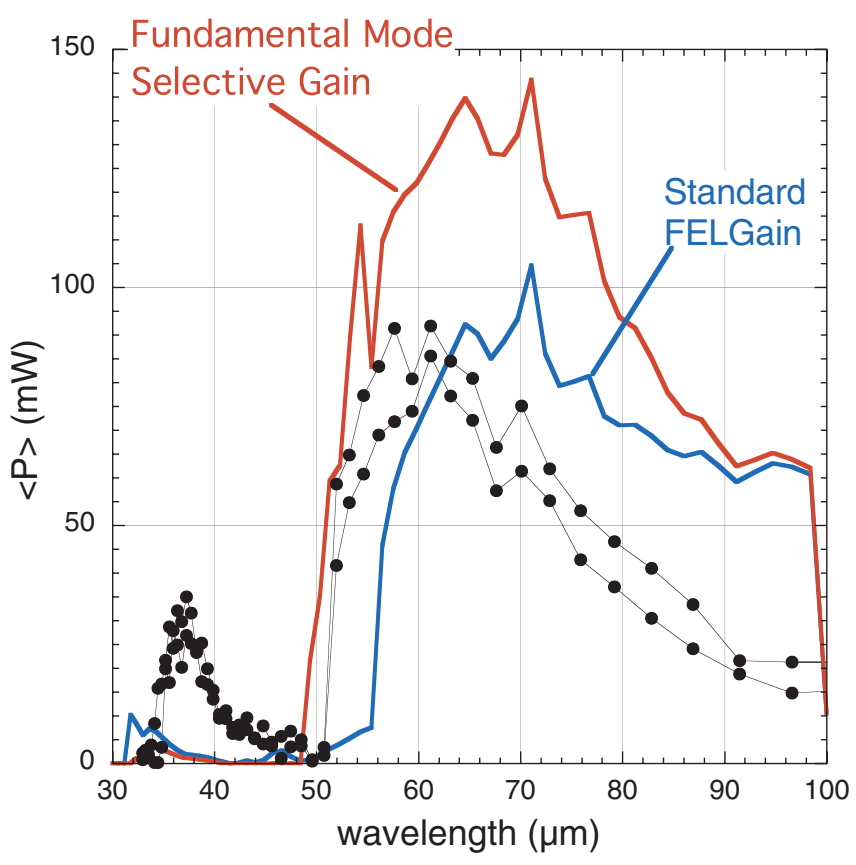

FIG. 4. (Color) Laser power vs centroid wavelength, for toroidal cavity, using hole coupling of $3 \mathrm{~mm}$. Bold lines are simulations.

waveguide aperture. It occurs only in the vertical plane, where the laser mode is guided.

At $\lambda_{b}=48.4 \mu \mathrm{m}$, about $20 \%$ of the laser energy is lost at the two entrances of the waveguide. It represents only $8 \%$ at $\lambda_{b}=53.3 \mu \mathrm{m}$. This explains the peak of losses in the curve of Fig. 5, which is also responsible for the spectral gap observed in Fig. 2.

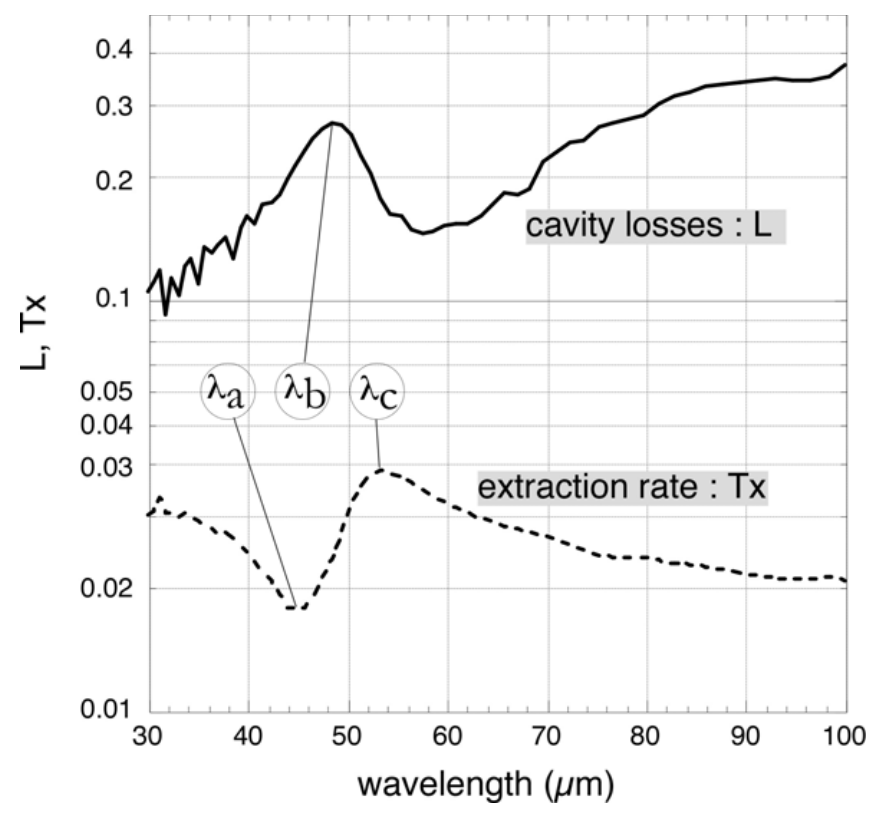

FIG. 5. Simulation of cavity losses $L$ and hole coupling extraction rate $T_{X}$ as a function of wavelength, for spherical cavity. 

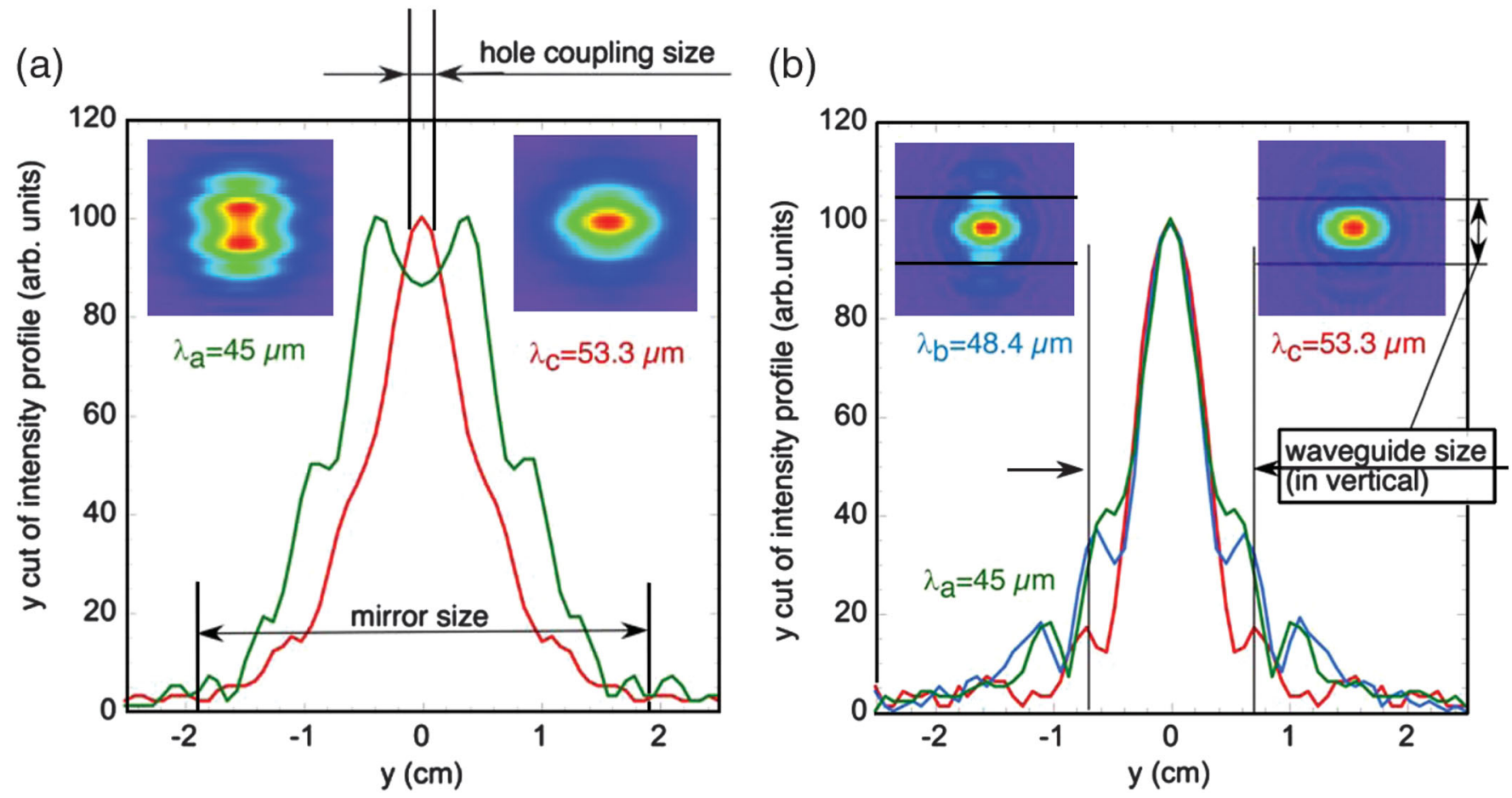

FIG. 6. (Color) (a) Numerical simulation in the case of spherical cavity: laser mode transverse profiles on the output mirror, for two different wavelengths. (b) Numerical simulation in the case of spherical cavity: laser mode transverse profiles on downstream waveguide entrance (point B in Fig. 1), for three different wavelengths.

\section{Simulation for toroidal cavity}

As shown in Fig. 4, the spectral gap phenomenon with a toroidal cavity is even stronger than in the case of spherical cavity (Fig. 2). Figure 7 shows the cavity losses $L$, and the hole coupling extraction rate $T_{X}$, as a function of wavelength, for a toroidal cavity. The red curve corresponds to simulation using fundamental mode selective gain, and the blue curve corresponds to uniform gain. The extraction rate exhibits a steep variation close to $50 \mu \mathrm{m}$, whose position is dependent on the eigenmode filtering of the gain (difference between red and blue dotted curves). The real case is in between these extreme configurations (see Sec. III B).

There is a large range, close to $40 \mu \mathrm{m}$, showing nearly zero extraction rate. Also, within this range, the losses are varying strongly, and exhibit an important minimum at $37 \mu \mathrm{m}$. Such small losses are creating a large intracavity power $P_{\text {in }}$, as shown in Fig. 8 which displays intracavity power $P_{\text {in }}$. In the simulation, this large value of $P_{\text {in }}$ is not sufficient to compensate the low extraction rate $T_{X}$, and output power $P_{\text {out }}=P_{\text {in }} T_{X}$ (see Fig. 4) remains close to zero. However, in real experimental conditions, a small misalignment of cavity mirrors or of the electron beam allows the large intracavity power of the laser mode to be better out coupled by the hole. In measurements, displayed in Fig. 4, this gives a significant output power $P_{\text {out }}$ at $\lambda=$ $37 \mu \mathrm{m}$.

The spectral gap, which is observed here in the range 40 to $55 \mu \mathrm{m}$, is therefore due to the conjunction of a mini- mum of the extraction rate $T_{X}$ with substantial cavity losses. Figure 9(a) displays the profiles on an output mirror (calculated with uniform standard gain, i.e., for blue curves). The "two spots shape" of the mode at $36.3 \mu \mathrm{m}$ explains the low extraction rate $T_{X}$ for $\lambda<56 \mu \mathrm{m}$. The

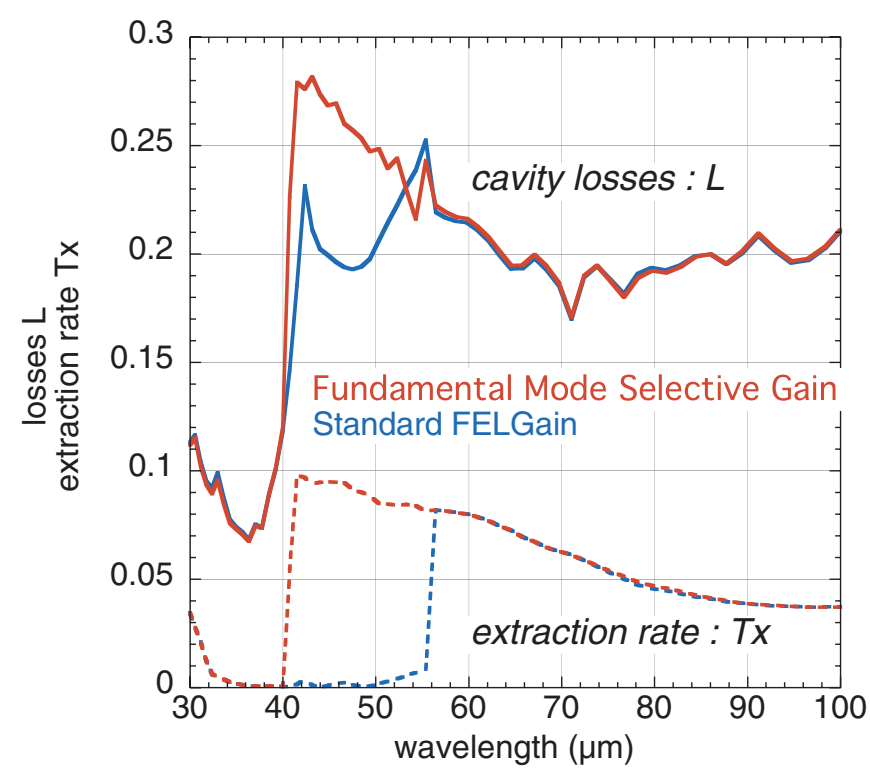

FIG. 7. (Color) Simulation, total cavity losses $L$ and hole coupling extraction rate $T_{X}$ as a function of wavelength, for toroidal cavity. 


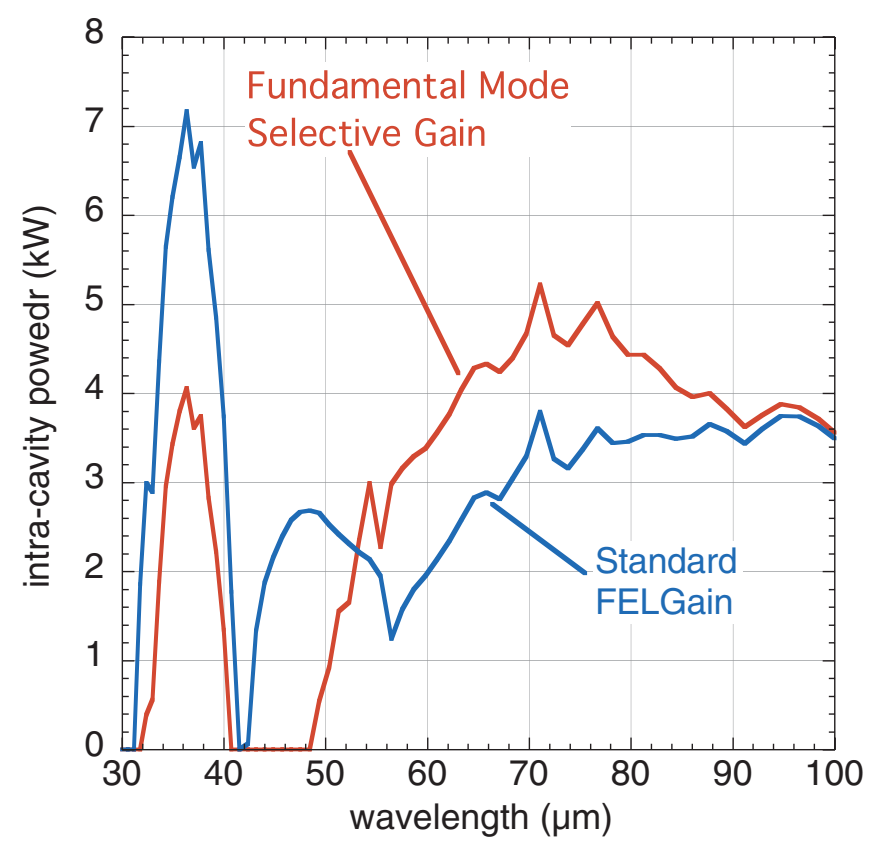

FIG. 8. (Color) Simulation, intracavity power $P_{\text {in }}$, as a function of wavelength, for toroidal cavity.

mode jumping, from 2 spots to 1 spot, between 55.4 and $56.5 \mu \mathrm{m}$, explains the sharp variation of $T_{X}$ at $56 \mu \mathrm{m}$. Also, the strong minimum of cavity losses at $36.3 \mu \mathrm{m}$ is due to the low extraction rate and the good fit of the mode profile into the waveguide aperture. Figure 9(b) displays the mode profiles at the downstream waveguide entrance (point B in Fig. 1). The sum of losses $L$ at both entrances represents only $2.6 \%$ at $\lambda=36.3 \mu \mathrm{m}$, which is rather low as compared to the other wavelengths.

\section{RESULTS FOR OTHER INFRARED FELS}

\section{A. Results for "FELIX"}

The optical cavity of the FELIX infrared FEL [3] is of the same type as for CLIO, but with minor differences: the downstream cavity mirror is in contact with the waveguide, i.e., there is no free space between that mirror and the waveguide entrance. On the other hand, there is a free space of $1.66 \mathrm{~m}$ in front of the upstream mirror. The distance between cavity mirrors is $6 \mathrm{~m}$, and the waveguide length is $4.34 \mathrm{~m}$, both of them are longer than CLIO. Figure 10 shows a comparison between simulation and measurement of the output power. Two measurements are displayed here: (1) for a perfect alignment of the cavity, and (2) for an alignment optimized at $\lambda=35 \mu \mathrm{m}$. It shows that the spectral gap phenomenon exists on FELIX. The depth of spectral gaps may change with FEL alignment, but their positions remain identical.

The simulation gives very similar results when using the "mode sensitive gain" or the "uniform gain." Simulations exhibit the two main spectral gaps, at 38 and $53 \mu \mathrm{m}$. We have done a detailed simulation around $\lambda=50 \mu \mathrm{m}$, which is shown in Fig. 11. It displays $P_{\text {out }} P_{\text {in }} L$ and $T_{X}$ as a function of wavelength, and shows the mode profiles on an
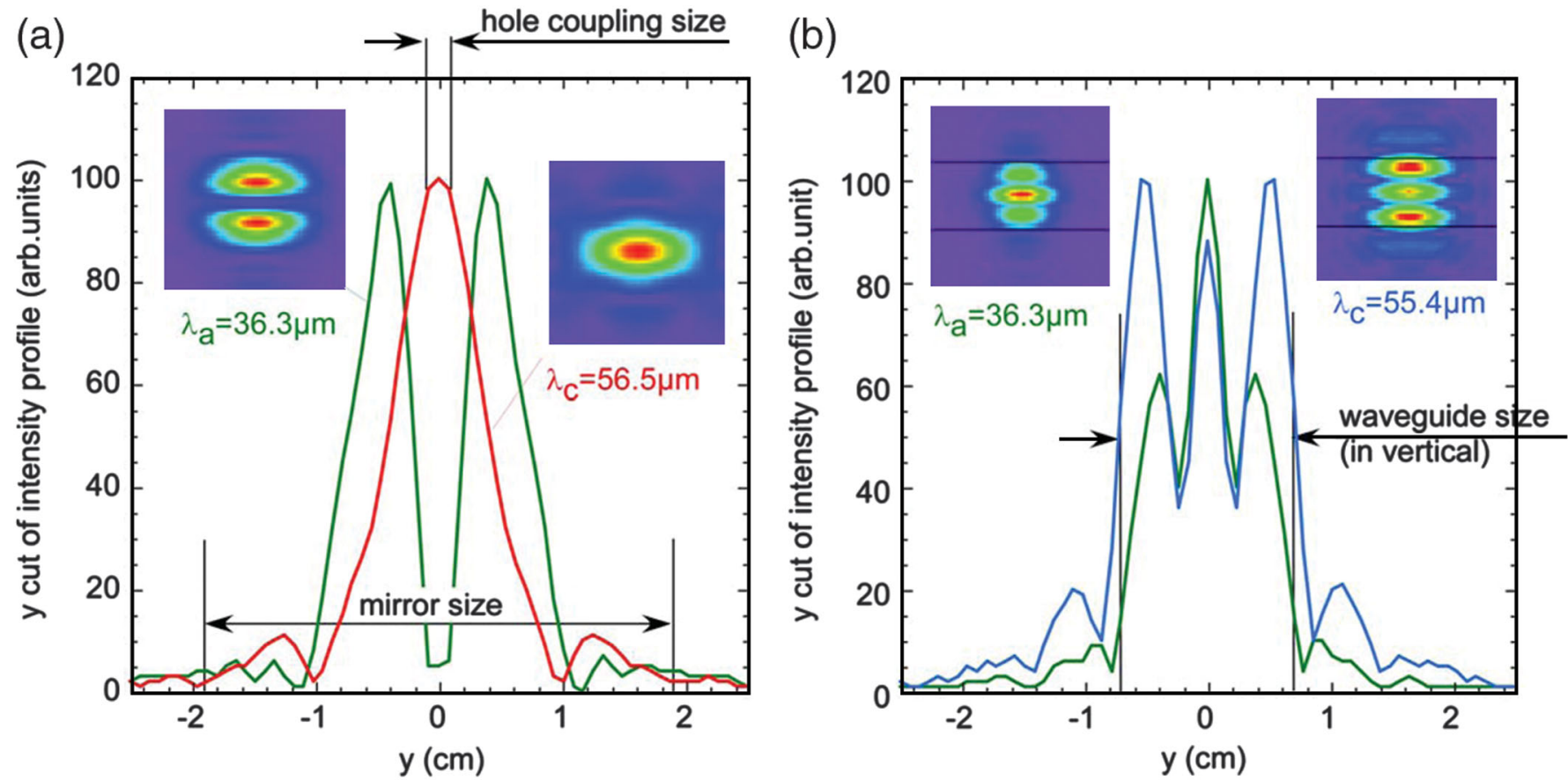

FIG. 9. (Color) (a) Numerical simulation in the case of toroidal cavity: laser mode transverse profiles on the output mirror, for two different wavelengths. (b) Numerical simulation in the case of toroidal cavity: laser mode transverse profiles on downstream waveguide entrance, for two different wavelengths. 


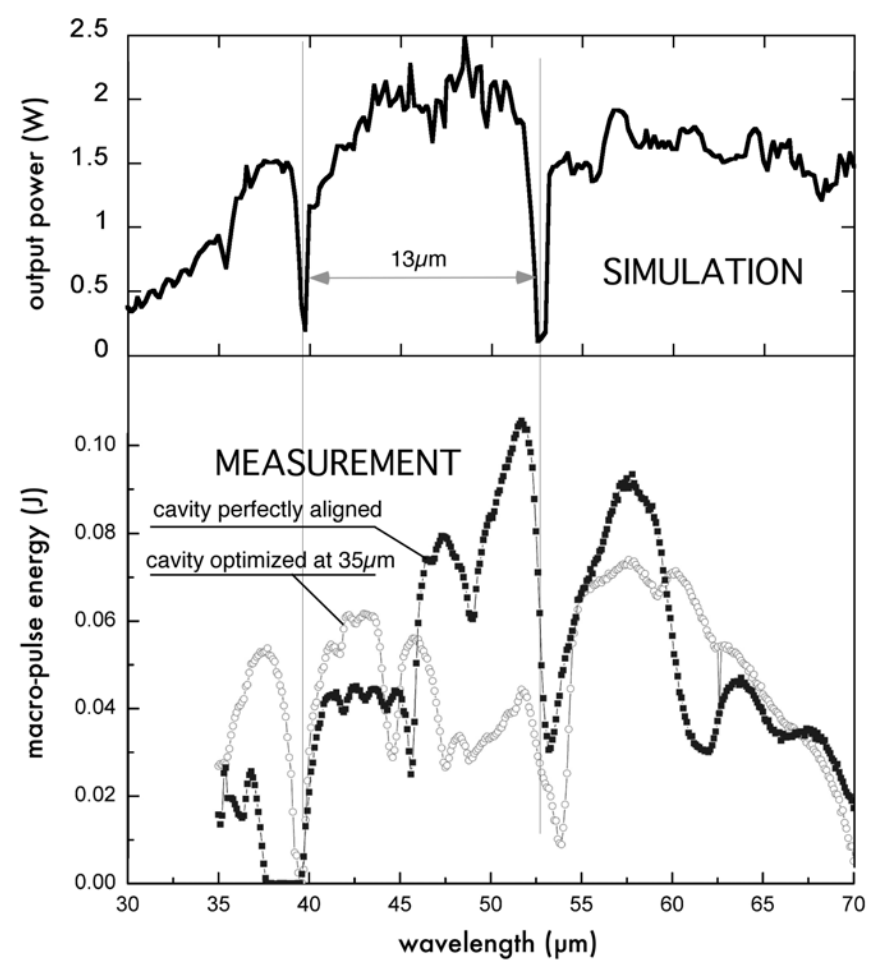

FIG. 10. Results for the FELIX infrared FEL.

output mirror (top images) and at the waveguide entrance (bottom images). The main reason of the spectral gap at $53 \mu \mathrm{m}$ is a minimum of extraction rate $T_{X}$ : The two spots shape of the laser mode profile on the output mirror has a minimum of intensity on axis. On the other hand, the change of mode shape near $50 \mu \mathrm{m}$ decreases cavity losses, inducing an increase of intracavity power $P_{\text {in }}$. However, this variation is dominated by the decrease of $T_{X}$ in the calculation of the output power $P_{\text {out }}=T_{X} P_{\text {in }}$. Note that for $\lambda \geq 53.4 \mu \mathrm{m}$, the mode exhibits a large transverse profile (in vertical), which does not fit the waveguide entrance. This explains the increase of the cavity losses.

As a summary, the spectral gap occurs at wavelengths corresponding to a jump of laser mode to a complex shape. Decreasing output power occurs when the laser mode exhibits a two spots shape on the output mirror, which creates a decrease of the coupling $T_{X}$. This special mode is induced by the waveguide constraints. Indeed, we will see in Sec. VI that these constraints are depending on the dimensions of the waveguide.

\section{B. Results for "ELBE"}

The configuration of the U100 FEL, at ELBE laboratory in Forschungszentrum Dresden Rosendorf, Germany, is very similar to the FELIX FEL: the waveguide is in contact with downstream mirror. But the optical cavity is much larger, 11.5 meters long, and the waveguide also, $7.9 \mathrm{~m}$. Figure 12 shows the result of the simulation, and displays the output power as a function of wavelength. The simulation gives very similar results when using the "mode

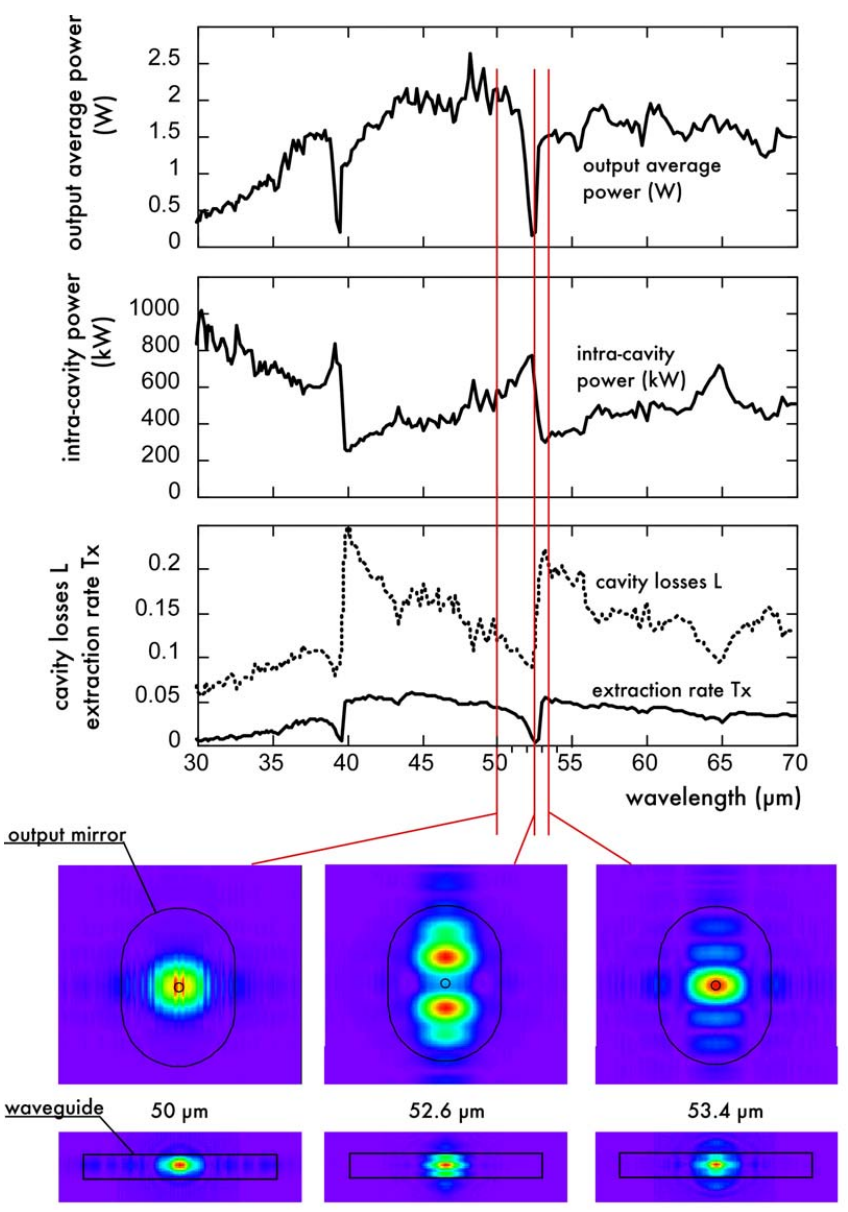

FIG. 11. (Color) Simulation for FELIX, with mode profiles on the output mirror and at the waveguide entrance, close to $\lambda=$ $50 \mu \mathrm{m}$.

sensitive gain" or the "uniform gain." Four spectral gaps are observed and they seem to be periodically spaced, with about a $7 \mu \mathrm{m}$ period. Unfortunately, the measurements of output power on ELBE are very lacunar, and they exhibit air absorption lines in the spectrum. Measurements in a dry air environment are planned in the future.

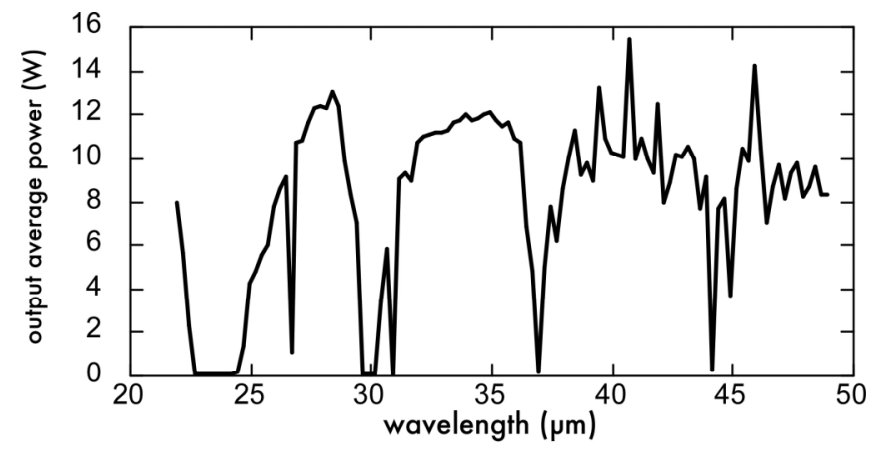

FIG. 12. Simulation of output power on the U100 FEL in ELBE, at $32 \mathrm{MeV}$, with $2 \mathrm{~mm}$ hole coupling. 


\section{PHASE ANALYSIS IN THE WAVEGUIDE}

The spectral gaps phenomenon is due to a particular combination of eigenmodes TEq and TMq, produced in the waveguide, which creates a mode profile producing large losses or low extraction rate. These eigenmodes are only vertically guided, because of the configuration of the waveguide (see Sec. II). Therefore the $p$ index of the modes is not used here. Though the numerical code does not use the eigenmodes to compute the wave propagation in waveguide section, we can deduce from the resulting profiles the energy distribution of the eigenmodes TEq and TMq in the waveguide. Figure 13 shows an example of this distribution in the case of CLIO with a spherical cavity at $\lambda=40 \mu \mathrm{m}$. The profile corresponds to downstream output of the undulator. The "mode selective gain operator" has been used here, with exclusive amplification on fundamental mode $q=1$. The energy on high order modes $q>1$ comes from coupling (see Sec. III B).

Even modes are not present here, because they have a zero amplitude on axis and are not amplified by the electron beam which is centered on axis. This figure shows that the most important modes are $q=1$ and $q=3$. It is likely that the phenomenon of spectral gaps is linked to the relative phase of the dominant cavity modes at the ends of the waveguide. Indeed, this phase will determine the diffraction losses at the entries of the waveguide and the

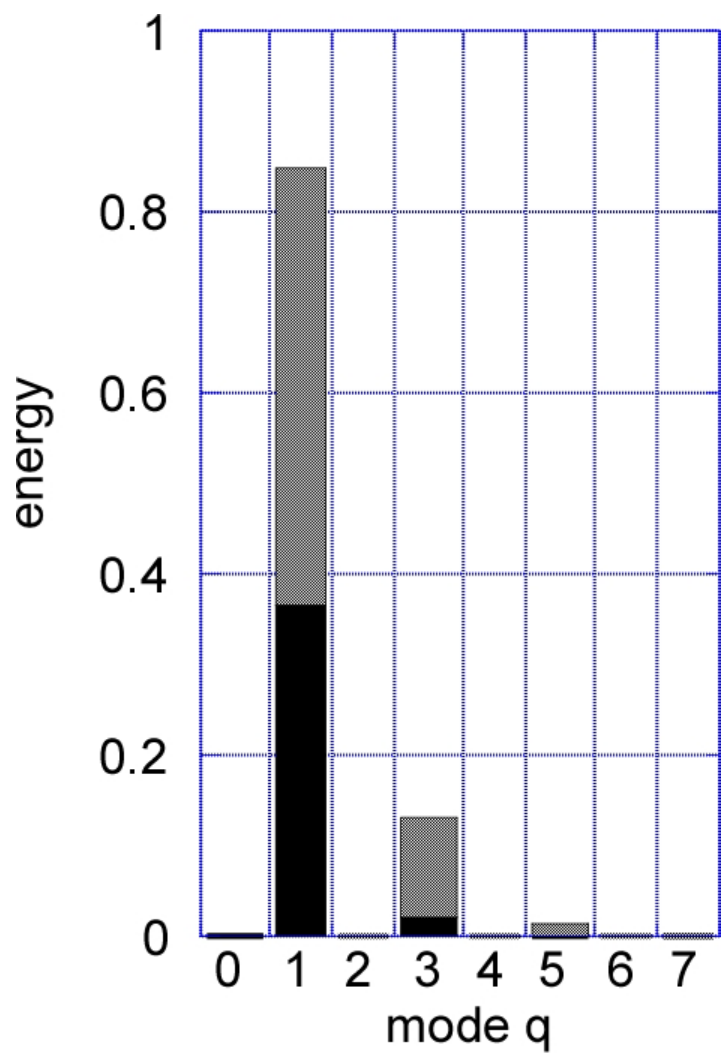

FIG. 13. (Color) Energy distribution of eigenmodes on TEq and $\mathrm{TMq}$ in the case of CLIO, with spherical cavity, at $\lambda=40 \mu \mathrm{m}$. mode structure at the extraction hole. Therefore, when sweeping the operating wavelength, one expects that a phase difference of $2 \pi$ in a cavity round-trip will account for the wavelength difference between two successive gaps.

The phase velocity in the waveguide for the mode $q$ is

$$
v_{q}=\frac{c}{\sqrt{1-\left(\lambda / \lambda_{c}\right)^{2}}} \cong c\left(1+\frac{\lambda^{2} q^{2}}{8 b^{2}}\right),
$$

where $\lambda_{c}=2 b / q$ is the cutoff wavelength, and $b$ is the waveguide aperture in vertical axis. The propagation time of mode $q$, in a waveguide of length $L$, is $t_{q}=L / v_{q}$. The relevant dephasing of mode $q$ is

$$
\phi_{q}=\frac{2 \pi}{T} t_{q} \cong \frac{2 \pi L}{\lambda}\left(1-\frac{\lambda^{2} q^{2}}{8 b^{2}}\right) .
$$

The phase difference $\Delta \phi=\phi_{1}-\phi_{3}$ between modes $q=$ 1 and $q=3$ is

$$
\Delta \phi=\phi_{1}-\phi_{3}=\frac{2 \pi L \lambda}{b^{2}} .
$$

For a complete cavity round-trip (RT):

$$
\Delta \phi_{\mathrm{RT}}=\phi_{1}-\phi_{3}=\frac{4 \pi L \lambda}{b^{2}} \text {. }
$$

This calculation does not make any hypothesis on the absolute phase distribution of the modes. It only gives the phase shift between mode 1 and mode 3 , for one cavity round-trip. Now, when choosing $\lambda$ equal to the wavelength of a spectral gap, then the "next" spectral gap in the FEL spectrum, at wavelength $\lambda^{\prime}>\lambda$, must keep the same phase structure. This is the unique hypothesis that we do here: the phase structure is the same for all spectral gaps (for a given FEL configuration, of course). Therefore, for

$$
\Delta \phi_{\mathrm{RT}}\left(\lambda^{\prime}\right)=\Delta \phi_{\mathrm{RT}}(\lambda)+2 \pi,
$$

one obtains

$$
\delta \lambda=\lambda^{\prime}-\lambda=\frac{b^{2}}{2 L},
$$

where $\delta \lambda$ is expected to be the wavelength difference $(\lambda-$ $\lambda^{\prime}$ ) between two successive gaps in the FEL power spectrum. Note that the $2 \pi$ periodicity in expression (5) corresponds to a $4 \pi$ periodicity when using upper modes $q=3$ and $q=5$. Therefore, as stated in the above hypothesis, the whole structure of modes TEq and TMq is kept constant between two consecutive spectral gaps at $\lambda$ and $\lambda^{\prime}$.

In order to check this simple analytical model, we show in Fig. 14 a simulation of FEL power as a function of wavelength, for various dimensions of waveguide aperture $b$. The parameters used in this calculation correspond to the ELBE free-electron laser, where the configuration gives the shorter wavelength period $\delta \lambda$.

The black lines correspond to spectral gaps, and the distance between two successive spectral gaps is $6.3 \mu \mathrm{m}$ 


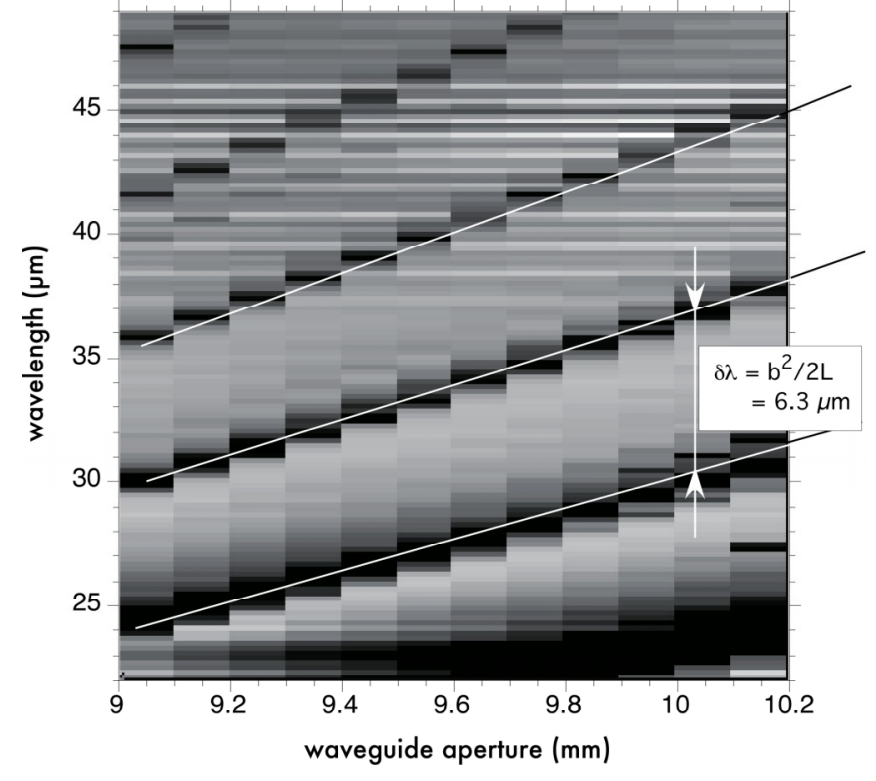

FIG. 14. Simulation-ELBE FEL power vs wavelength and waveguide aperture $b$.

as predicted by the simple analytical expression (6) with $b=1 \mathrm{~cm}$ and $L=7.92 \mathrm{~m}$. Therefore, the simple model and the simulation agree very well, even if the available data do not allow one to verify it experimentally.

Now for FELIX, the expression (6) gives $\delta \lambda=$ $11.5 \mu \mathrm{m}$, with $b=1 \mathrm{~cm}$ and $L=4.34 \mathrm{~m}$. The simulation curve in Fig. 10 shows that the distance between two successive spectral gaps is $\delta \lambda=13 \mu \mathrm{m}$, which is not far from analytical and experimental values.

For the case of CLIO, the expression (6) gives $\delta \lambda=$ $50 \mu \mathrm{m}$. The first spectral gap in the spectrum is measured at $\lambda=45$ to $50 \mu \mathrm{m}$. Therefore, the next spectral gap should be observed close to $\lambda=100 \mu \mathrm{m}$, which is not the case. This could be explained by a further refinement of the above analytical model: one can assume that the gap occurs when the phase difference between mode 1 and 3 is $(2 n+1) \pi$, with $n$ integer, along one pass in the waveguide. Indeed, if the phase between the two modes is such that the electric fields add at the center of the chamber and subtract on the sides, the intensity profile will exhibit the smallest possible spatial width, thus minimizing diffraction losses. Also, the profile peaking at the center will favor laser power extraction by the out coupling hole. If the phase difference along the chamber is $2 n \pi$, one gets the same profile at each end, which is the more desirable situation and if it is $(2 n+1) \pi$, it is the most disadvantageous one, giving rise to the spectral gap. Expression (6) becomes then

$$
\lambda=\frac{b^{2}}{2 L}(2 n+1) .
$$

This accounts for the position of the first gap, then at $50 \mu \mathrm{m}$ on CLIO, as observed, and the second should be at $150 \mu \mathrm{m}$, where diffraction losses are too large to obtain lasing. At FELIX and ELBE, one side of the waveguide is in contact with one of the cavity mirrors, so that the effective length of the waveguide is doubled and Eq. (6) becomes

$$
\lambda=\frac{b^{2}}{4 L}(2 n+1)=\frac{b^{2}}{2 L}\left(n+\frac{1}{2}\right) .
$$

The periodicity remains unchanged and the first gap is situated at a short wavelength $(<10 \mu \mathrm{m})$ where no waveguiding occurs. The conditions expressed in (7) and (8) are compatible with Eq. (6), they are only more restrictive.

This explanation is not fully quantitative, as already mentioned above. In particular, it does not explain the "violent" character of the increase of the losses in the vicinity of the spectral gap. As can be seen on Fig. 11, the optical mode shape exhibit two peaks only in a short wavelength interval around the one where the minimum power occurs. This is due to fast variations in the content of waveguide modes, not taken into account in the simple analytic model but only in the simulations.

Nevertheless, the above expressions provide an order of magnitude of the number $N_{\mathrm{PG}}$ of spectral gap in a given FEL spectral range, proportional to $L / b^{2}$.

\section{CONCLUSION}

The spectral gap phenomenon has been observed on various infrared FELs. This phenomenon produces a series of holes in the tuning range of the FELs using partial waveguiding of the light, in order to alleviate diffraction losses in the far infrared. It is unavoidable and depends only on the characteristics of the waveguide used. The explanation of the spectral gap phenomenon is quite complex, because it is dependent on two independent parameters: (1) hole coupling extraction rate, and (2) optical losses in waveguide entrance. Both of them are strongly dependent on slight variations of the transverse mode profile. Also, they can be somewhat uncorrelated, as shown in Fig. 7, leading to large differences in the behavior of the gaps. As a consequence, a simple analytical model, described above, only gives an order of magnitude of the expected number of spectral gaps in the FEL range. A detailed analysis requires the simulation code, whose results are in rather good agreement with measurements.

We have shown here that the number of spectral gaps is proportional to the waveguide length and to $1 / b^{2}$, where $b$ is its width. In the typical tuning range of an infrared FEL the number of gaps can be quite large. This is a very penalizing problem for a FEL, whose main virtue is normally its large range of tenability. This must be taken into account in the engineering of future far-infrared FELs using partial waveguiding in the optical cavity. 


\section{ACKNOWLEDGMENTS}

We are thankful to Lex Van der Meer, and more generally to the FELIX free-electron laser group, for their contribution by sending us experimental data for the FEL. Thanks also to W. Seidel from the ELBE freeelectron laser for sending data from the FEL.

[1] M.E. Couprie and J. M. Ortega, Analusis 28, 725 (2000).

[2] J. M. Ortega, F. Glotin, and R. Prazeres, Infrared Phys. Technol. 49, 133 (2006); R. Prazeres, F. Glotin, and J. M. Ortega, Nucl. Instrum. Methods Phys. Res., Sect. A 528, 83 (2004), http://clio.lcp.u-psud.fr/clio_eng/clio_eng.htm.
[3] Li Yi Lin and A. F. G. van der Meer, Rev. Sci. Instrum. 68, 4342 (1997), http://www.rijnhuizen.nl/research/guthz/ felix_felice/.

[4] P. Michel et al., in Proceedings of FEL 2006-BESSY, Berlin, Germany, pp. 488-491, http://cern.ch/AccelConf/ f06/PAPERS/TUCAU02.PDF.

[5] R. Prazeres and V. Serriere, Nucl. Instrum. Methods Phys. Res., Sect. A 475, 524 (2001); R. Prazeres, Eur. Phys. J. Appl. Phys. 16, 209 (2001).

[6] R. Prazeres, F. Glotin, and J. M. Ortega, Eur. Phys. J. Appl. Phys. 29, 223 (2005).

[7] G. Dattoli, J. Appl. Phys. 84, 2393 (1998); G. Dattoli and P. L. Ottaviani, Opt. Commun. 204, 283 (2002); G. Dattoli, T. Letardi, J. M. J. Madey, and A. Renieri, Nucl. Instrum. Methods Phys. Res., Sect. A 237, 326 (1985).

[8] J. W. J. Verschuur, G. J. Ernst, B. M. van Oerle, and D. Bisero, Opt. Commun. 133, 229 (1997). 